\title{
ACTIVE LEARNING THROUGH TECHNOLOGICALLY-BASED DECISIONS
}

\section{Tsvetelina Ivanova ${ }^{1}$}

\begin{abstract}
The fourth industrial revolution determined Education 4.0, which brings out both the clear presence of technology in the educational process and connects electronic devices in the real and virtual world during learning, and during activities of interest, according to personal preferences and experience. However, the requirements and conditions for the use of technologies to increase learning outcomes are much more important. The objective of the study is the construct "technologybased decision making". The research aims to study the influence of the technology-based decisions for the active learning of the students from the higher educational institutions /HEIs/. A scientific study was conducted with 74 students from HEIs. The results show that technology-based decision-making increases learning activity and provides opportunities for learning to be a constructive and active process. Technology-based decisions of university students are defined as a specific motivator for their active learning.
\end{abstract}

UDC Classification: 378, DOI: https://doi.org/10.12955/pss.v2.213

Keywords: learning, tech-decision, Ed-Tech-decision

\section{Introduction}

Each student from the higher educational institutions /HEIs/ uses virtual devices in their daily lives and for learning. "Technology has become an irreversible force driving the transformation of teaching and learning practices" (Cheung et al., 2021). The construct "technology-based decisions" is defined as decisions related to using a particular technology as more suitable than another technology as a whole or its individual functionalities for a particular life or educational activity. Technology-based decisions determine the creation of smart learning environments /SLEs/. "The SLEs not only consists of intelligent tools, learning resources, learning community and teaching community, but also the learning ways and teaching ways, which make the learning environments "smart" according to the different needs of learning“" (Ronghuai et al., 2013). In implementing Education 4.0, ubiquitous learning and intelligent learning environments create conditions for HEIs students to make technology-based decisions for active learning. While traditional learning provokes passive learning, active learning requires "seeking new information, organizing it in a way that is meaningful, and having the chance to explain it to others." (Allen \& Tanner,2005).

Today's generation of students from HEIs lives and studies in and through the Web. In this context, they have to make technology-based decisions daily, including for active learning. Active learning is already associated with active and independent decision-making by learners."Active learning requires students to think hard and practice using new knowledge and skills to develop long-term recall and a deeper understanding. This deeper understanding will also enable learners to connect different ideas together and to think creatively, once the initial knowledge base is secure." (Active learning, 2020). When using the "technology-based decisions" construct in education, the "EdTech-decision" construct is used.

The technology-based decisions for the educational process made by the students from HEIs are prerequisites for satisfaction with the learning process. Each student chooses a subjectively preferred electronic device, subjectively preferred technologies for more effective learning, subjectively preferred technologies for improving the outcomes from education. In this context, technology-based decision-making to support the active learning of students by HEIs can find explanatory models through the rational decision-making model (Edwards, 1954); through the "garbage model" for decision making (Cohen et al., 1972); through the multi-criteria decision-making model (Hollands \& Escueta, 2017).

This research aims to study the impact of technology-based decisions and determine if they positively motivate HEI students to learn actively.

In this context, the scientific questions that are asked are:

- how students from HEIs make technology-based decisions for their education;

- the extent to which technology-based decisions motivate HEIs students to learn more effectively and actively.

\footnotetext{
${ }^{1}$ Plovdiv University „Paisii Hilendarski“, Faculty of physics and technology, Educational Technologies, Bulgaria, tsvetelina.1@uni-plovdiv.bg
} 


\section{Methodology}

The semi-structured interview included questions about HEIs students active learning, technologybased decision-making in the active learning process, and the impact of technology-based decisions on active learning by HEIs students.

A questionnaire with 29 items included, which are composed of four groups:

- For satisfaction with distance learning in e-environment and the answers from which the participants choose in the survey are "yes" and "no";

- To use active learning methods in an electronic environment remotely with open answers;

- For making technology-based decisions for different aspects of the learning process: in teaching, in solving independent tasks, in the process of teamwork with colleagues, in performing exam tasks, for the administrative status. The answers to 7 of the items are with a choice of "yes" or "no", and for 8 of the items are open answers if offered.

- To increase motivation to learn through the use of technology and technology-based decisions, with "yes" and "no"“.

The survey was developed and implemented through Google Form, which was provided to HEIs students.

\section{Participants in the study}

The study was offered to 120 students from the University of Plovdiv and Sofia University, with the voluntary participation of 74 students.

\section{Procedure}

A semi-structured interview was first conducted with the study participants in Google meet on the content of the concept of "technology-based decisions", "active learning" and the role of technologybased decisions for active learning. Examples and case studies on the impact of technology-based solutions on active learning are discussed. After the semi-structured interview, participants complete the survey using the code sent to them from Google Form. The code was sent via the Google meet chat and via the social network Facebook.

\section{Results}

\section{Results from the semi-structured interview}

In the semi-structured interview, $79.72 \%$ of the participants in the study shows that they use in their education methods of active learning such as methods for problem-based learning, methods for project-based learning, methods for learning through research, methods for learning by doing/ practicing, various interactive methods, etc.

Regarding the use of technology-based decisions in support of the active learning methods they use in their training, $83.8 \%$ of the respondents answered that their motivation for active and independent learning increases through the application of technology-based decisions. The respondents understood the content of the construct "technology-based decisions" and gave the following answers: self-finding electronic resources as scientific literature $-68.8 \%$ of the respondents; independent and active development of presentations, independent and active development of schemes, diagrams, algorithms with Microsoft products - $43.6 \%$ of the respondents; author's development of lessons, worksheets, flashcards, etc. or development with own creative solutions and use of pictorial images from the Web in compliance with copyright requirements - $83.8 \%$ of the respondents; own choice of electronic platforms for discussions and joint learning with HEIs co-students - 31.3\% of the respondents; use of preferred software for solving learning tasks $-33.3 \%$ of the respondents.

The overall response rate in the semi-structured interview exceeds $100 \%$ because the respondents are entitled to more than one answer, as is usually the case in semi-structured interviews.

\section{Results of the survey}

All study participants responded to all of the items in the first group.

In the first group of items, the predominant percentage of answers for lack of technological difficulties is explained by the higher technological competencies of young people and the assessment by the teachers of the technological problems of the students from HEIs. In the second group of items related to active learning methods, the answers are open. The results with a positive answer show that during 


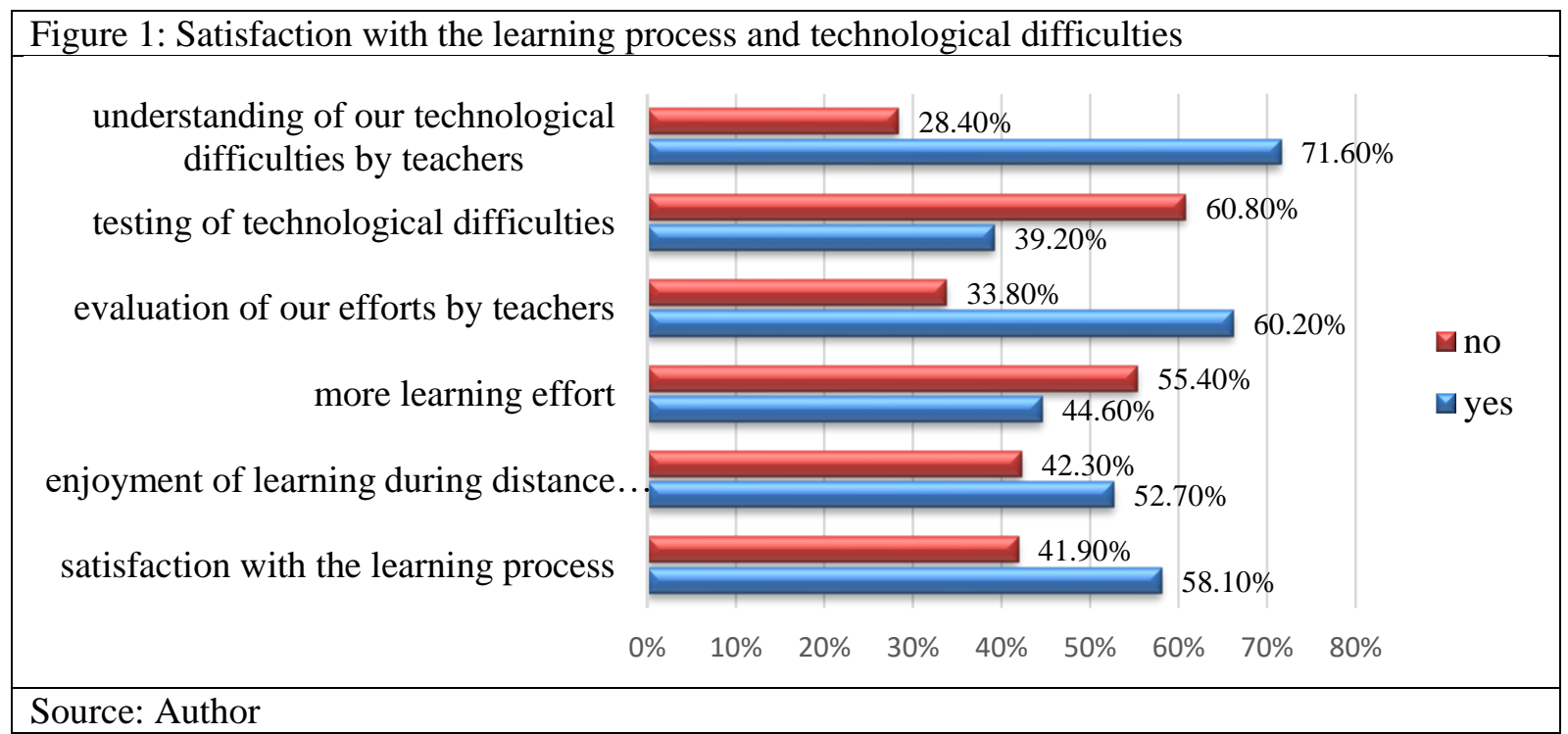

distance learning in e-environment, $33.8 \%$ of the respondents use project-based learning, problembased learning $-29.7 \%$, "learning by doing/through practice" $-25.7 \%$, research methods in teaching $33.8 \%$, the method of "inverted teaching" - $16.2 \%$, teamwork with fellow students $-54.1 \%$ with 20 variations of answers using electronic platforms Google Meet, Google Classroom, Microsoft Teems, Zoom, Judt Meet, Jitsi Meet, Skype, Discord meet. It is noteworthy that answers such as Google, online media, Wikipedia, ABI/Inform were also noted.

The answers to the third group of items show that electronic platforms that require technology-based decisions are repeated, but there are also new answers related to Microsoft products such as PowerPoint or online courses such as MOOCs or MuseScore software. At the same time, 59.2\% of respondents believe that technology increases their learning outcomes.

In summary, the making of technology-based decisions for education is estimated as a fact by $31.1 \%$ of respondents. The result obtained could find an explanatory model in the notion of a decision attitude: "the desire to make or avoid decisions, independent of any consequence that they achieve" (Beattie et al., 1994). Decision avoidance manifests itself as a tendency to avoid making a choice by postponing it or by seeking an easy way out that involves no action or no change. Decision avoidance is not the same as procrastination, although there is relevant research on decisional procrastination, an individual difference in the tendency to defer decisions (...) One may simply be maintaining energy for a later time rather than deliberately avoiding a decision" (Anderson, 2003). The second principle

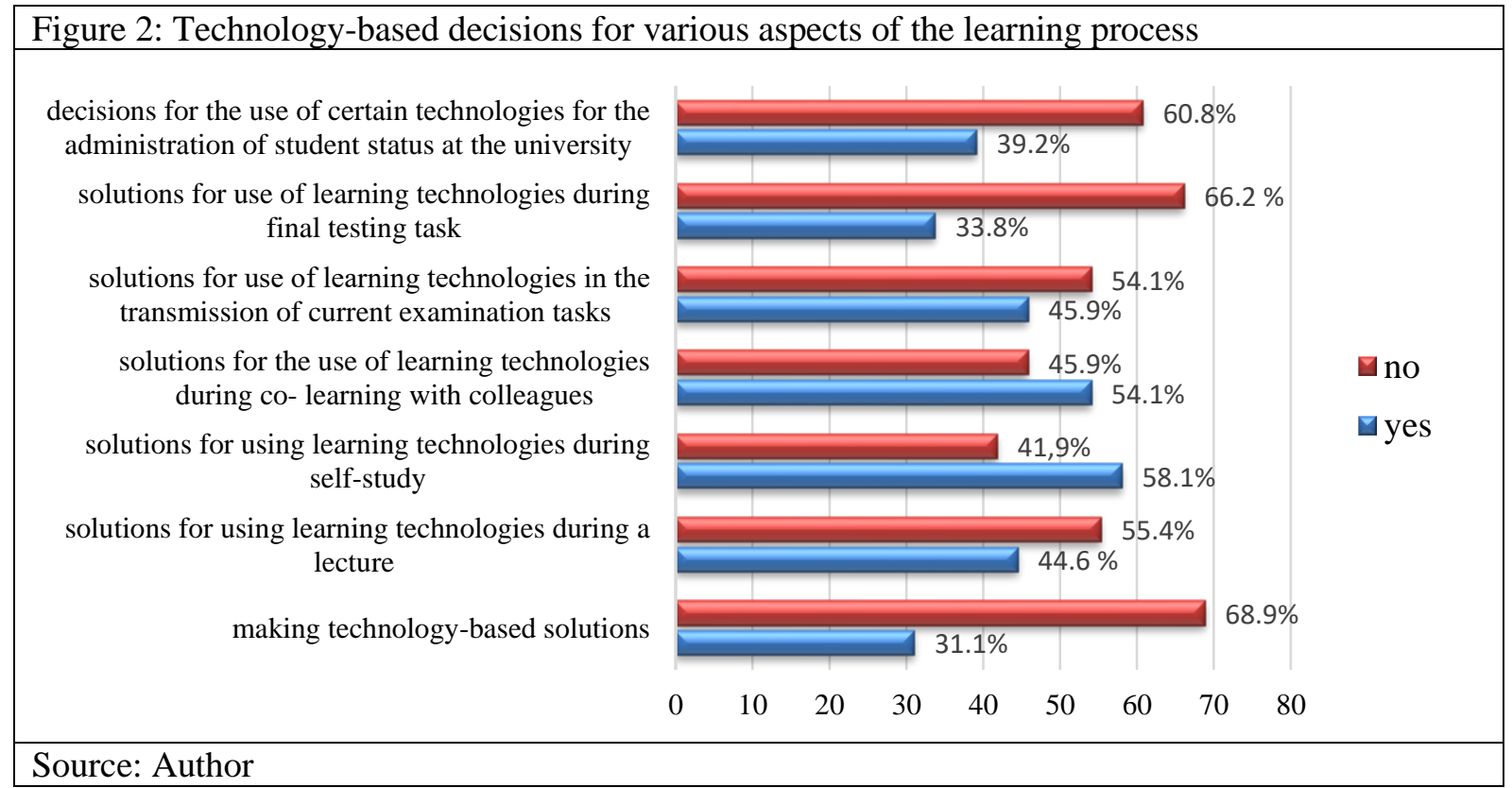


concerns the relevant antecedents of decision avoidance and is termed multiple causation. It is reasonable to consider that a complex and common behavior, such as decision avoidance, may have more than one cause. It also is likely that many observed antecedents of decision avoidance have their influence through mediating variables (Anderson, 2003).

The fourth group of items is related to the specifics of technology-based decision making. Respondents note ways to make technology-based decisions. The results are presented in Figure $3 \mathrm{a}$. and Figure $3 b$.

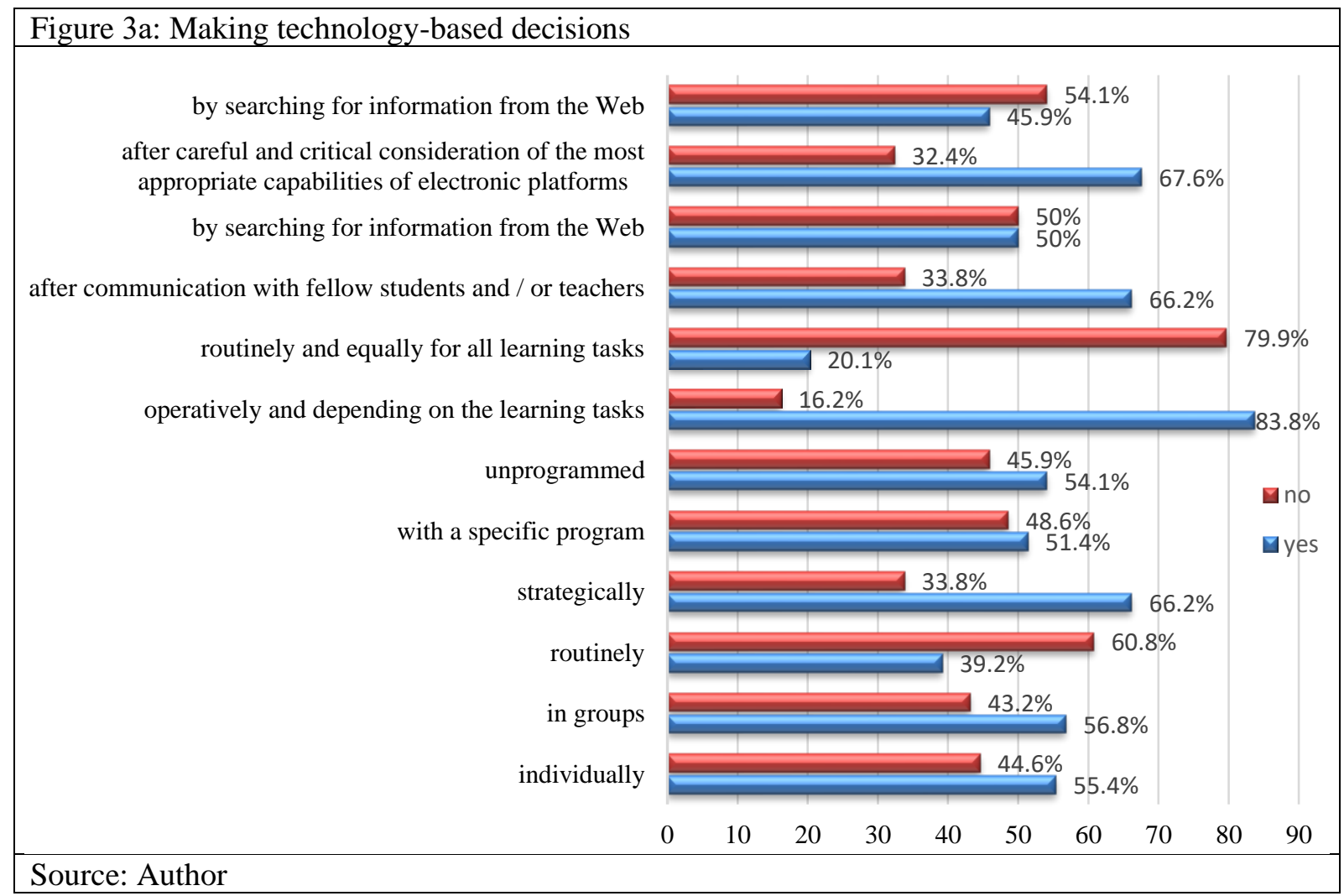

The answers to the individual items are more than $100 \%$ because the respondents can choose several answers. The received answers suggest that learning tasks are already available, there are already learning tasks for which a technological decision has been made repeatedly, and the respondents are clear that a certain type of tasks is performed most effectively with a certain type of technological decision, but they also have new learning tasks that require choice of technology or other technologically new decision. Another explanatory model can be deduced in the context of Iyengar \& Lepper (2000). According to them "Perhaps it is not that people are made unhappy by the decisions they make in the face of abundant options but that they are instead ensure--that they are burdened by the responsibility of distinguishing good from bad decisions" At the same time, respondents strive to

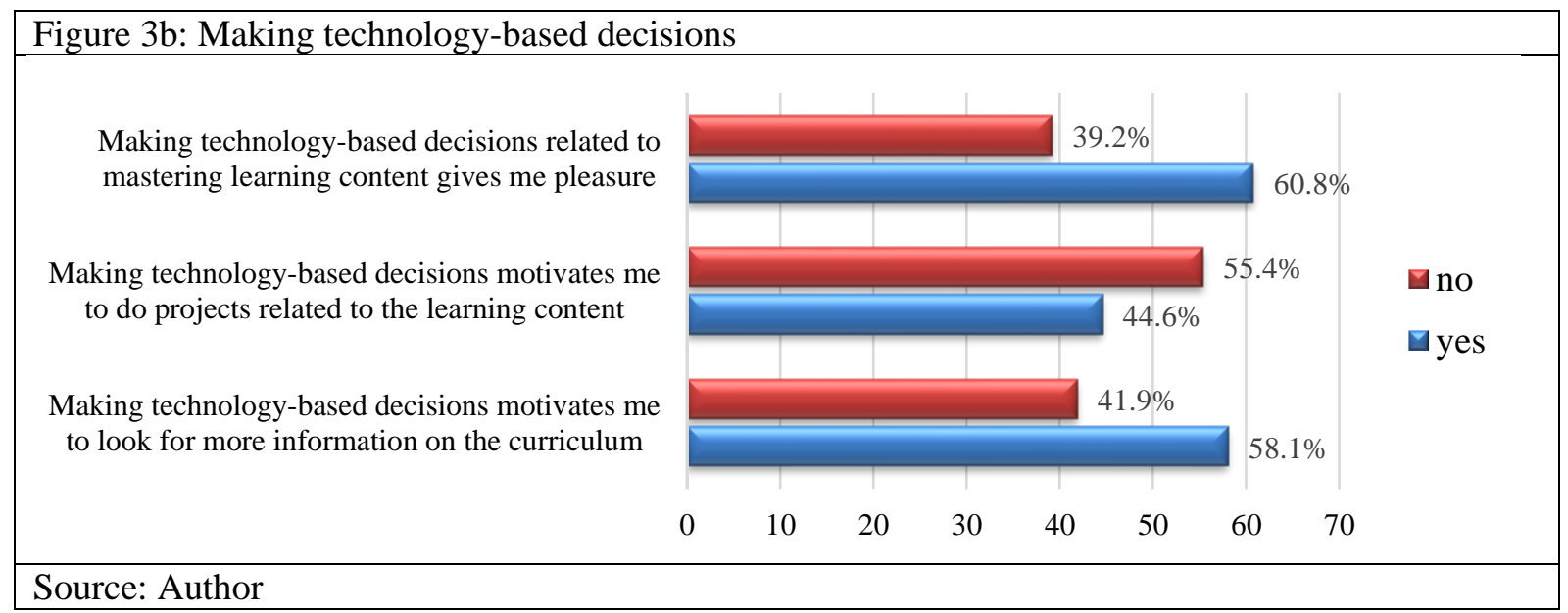


make strategic technological decisions (66.2\%), i.e. to cover more learning resources and refine decision-making according to the long-term consequences. The differences in the respondents' answers for making an individual (56.8\%) and group technological decisions (55.4\%) are minimal. This minimal difference in the results shows that the respondents have established routine learning task.

In Figure 3a, it is noticeable that there is a double answer for making technology-based decisions by searching for information on the Web with different respondents' answers. There is no repetition or inaccuracy, or ambiguity. The item "search for technology-based solutions on the Web" $(50 \%)$ is associated with the item "communication with fellow students and teachers" (66.2\%), as well as the item "after careful and critical consideration of the most appropriate capabilities of electronic platforms " $(67.6 \%)$. Respondents rely on information from the Web more for technology-based selflearning decisions than for communication. A fact that shows that the technology-based solutions of the respondents are already presented as a separate construct for independent and active learning.

As it can be seen from Figure 3a, the choice and making of Ed-Tech-decision influence the mastery of the learning content in three aspects: enjoyment of learning (60.8\% of respondents); project-based learning (in $44.6 \%$ of respondents); active and constructive learning (58.1\% of respondents). Making technology-based decisions by students increases the level of their active learning, both individually and as a team and group. Obviously, the technology-based decision-making construct has the strongest impact on the enjoyment of learning. When there is enjoyment in learning, then learning becomes imperceptible and desirable. The pleasure of learning motivates learning. The "technology-based decision making" construct does not sufficiently motivate the respondents to implement projects related to the learning content. Probably, the respondents do not find a clear connection of the educational content with its application in practice through technology-based decisions. The respondents' answers manifest an increase in the active and additional search for information on the learning content when they use technology-based decisions. The results show unequivocally that the making of technology-based decisions by the respondents has a positive effect on their active learning.

\section{Discussion}

The forced pandemic conditions for the ubiquitous digitalization of education and the development of Education 4.0 as a result of the development of the World Wide Web are increasingly requiring learners to make technology-based decisions for the realization of active learning.

Respondents predominantly note that they do not experience technological difficulties in the learning process or rely on teachers, colleagues or information from the Web on how to overcome the technological problems that have arisen. These results are not surprising for several reasons. On the one hand, the current generation of HEI students use electronic devices on a daily, almost constant basis and are on the World Wide Web, which allows them to master new functionalities of the used technologies much easier. On the other hand, the constant use of technology forms technological culture and digital literacy and the digital identity of each student. Digital identity is associated with the digital traces left by any digital search, digital post, or digital behavior. Each of these elements results from a technology-based decision, whether the decision was impulsive, spontaneous, or deliberate or sought a final non-technology-based decision. In the learning process, spontaneous and impulsive technology-based decisions are not predominant but considered and refined, probably due to the assessment of learning achievements and group control in the performance of the group or team tasks. In the learning context, digital footprints are less. In contrast, digital traces in each student's daily functioning are more frequent and sometimes impulsive, following the rules of daily communication. Regardless of the learning process or everyday life, every digital trace is associated with a technology-based decision for active learning.Technologically based decisions are much more structured when using different methods for active learning such as project-based learning, problembased learning, learning by doing, research methods, the method of inverted teaching. Although, according to the respondents' answers, the use of active learning methods the results vary from $16.2 \%$ to $33.8 \%$, each application of an active learning method requires not only one technology-based decision. The result obtained for learning enjoyment as a result of making technology-based decisions $160.8 \% /$ shows that non-technology-based decisions are increasingly giving way to technology-based decisions. Even with complete non-technology based decisions, digital traces of the required information can be found. Although respondents note the predominant answers for technology-based desicions in active learning are targeted at different types of e-learning platforms, and the learning 
organization or the teacher predominantly chooses the e-platforms, each respondent takes technologybased decisions for learning individually. Marked open answers for text editors such as (Word), presentation programs (Powerpoint, Prezi), e-textbooks or e-courses (MOOSC, Google search engines, online media, Wikipedia, and even ABI/Inform) are indicative that respondents seek and use other technology-based decisions that the training organization or teacher does not specify. From the respondents' answers, it is clear that they know and use not only basic programs from the Microsoft package or Google, but also my actions on Microsoft Windows, macOS, Android, iOS, Windows Mobile, etc. The knowledge of different electronic platforms, applications, programs allows operationalizing the technology-based decisions according to different criteria and variations, according to the set learning goals, according to the desired and perspective learning achievements in the process of active learning.

The operationalization of the technology-based decisions can be realized according to participation during lectures, individual and/or group work during exercises, implementation and delivery of current educational and final exam tasks. The variations of the respondents' answers for this type of operationalization of technology-based decisions are from $31.1 \%$ to $58.1 \%$, i.e. there is still insufficient differentiation of technology-based decisions for learning purposes.

Another possible operationalization model of technology-based decisions is seen in the results related to certain programs or unprogrammed, individual or group, strategic or operational, etc.

Strategic decisions in the learning process are subject to certain learning strategies, obviously personalized learning strategies are used, as well as possibly legal, albeit individual SWOT analysis. Respondents assess or have assessed the strengths and weaknesses of a technology-based decision, its advantages and capabilities, threats, and impact on implementing a certain type of learning tasks. In this context, the answers for making technology-based decisions with a certain program $(51.4 \%$ of the respondents) is almost equal to non-programmed making of technology-based decisions $(54.1 \%)$. Considering the stages of strategic decision-making, respondents assess the problem and the situation with learning tasks, construct decisions, choose options and implementation according to the chosen option, exercise control over implementation, and undoubtedly control over the chosen technological decision. Thus, routine technology-based decision-making is reduced to $39.2 \%$ of respondents, but we should not miss the fact that some of the strategic and unprogrammed decisions that have proven their effectiveness move to technological decisions with a specific program and become routine. Routine technology-based decisions reflect both the technological experience of the respondents and their experience in making decisions and making choices.

In this model of operationalization of technology-based decisions, the cognitive-motivational aspects of each student and the individual groups are important. The results demonstrate a clear motivation for learning among the respondents. The explanatory model could be sought in the direction of successful predictive implementation, given that the motivation corresponds to the abilities and capabilities of the respondents to use different virtual technologies. The available abilities and capabilities of the respondents are reflected in their opinions on the items from group 1 and group 2. In the presence of self-efficacy, respondents are motivated to put more effort and set goals that they believe they will be able to achieve.

The results show self-assessment of one's abilities and motivation to learn, which allow for higher learning achievements. On the other hand, learning and mastering the learning content has a personal meaning and personal significance for the respondents and, accordingly, motivation increases. Respondents' decision-making is perceived as self-control over technological circumstances and confidence that they themselves can effectively use technological devices and technological functionalities on various platforms and programs.

The two models of operationalization of technology-based decisions allow applying training models for making effective technological decisions in order to have higher efficiency of the educational achievements of the students from HEIs.

\section{Conclusion}

The results show that the construct "technology-based decision" making increases learning activity and provides opportunities for learning to be a constructive process. The making of technology-based decisions by students is a motivator for active learning. In the process of making technology-based 
decisions for the implementation of active learning, all three decision-making models are applied with the strongest presence of the rational model, followed by the model for multi-criteria decision-making and with the least presence of the garbage model of decision-making. The derived sequence indicates that the respondents' technology-based decisions are a specific motivator for their active learning. In this context, the implementation of training of students from HEIs to operationalize technology-based decisions would facilitate the learning process and improve their learning achievements. Through effective Ed-Tech-decision, HEIs students would not only realize an effective and constructive process of active learning, but the educational process itself in the opposite position of a boomerang would provoke creativity, critical thinking and more effective communication and cooperation in group and team activities. Technology-based decisions are important and significant for the active learning of students in a university environment and require future research

\section{Acknowledgments}

The research has been financed by Diversasia project 618615-EPP-1-2020-1-UK-EPPKA2-CBHE, funded by Erasmus+.

\section{References}

Allen D., Tanner K. (2005). Infusing active learning into the large-enrollment biology class: seven strategies, from the simple to complex. Cell Biol. Educ 4, 262-268.

Anderson, C. (2003). The Psychology of Doing Nothing: Forms of Decision Avoidance Result From Reason and Emotion. Psychological Bulletin. 129, 1, 139-167.

Beattie, J., Baron, J., Hershey, J. C., \& Spranca, M. D. (1994). Psychological determinants of decision attitude. Journal of Behavioral Decision Making, 7, 129-144.

Cheung, S.K.S., Kwok, L.F., Phusavat, K. et al. (2021). Shaping the future learning environments with smart elements: challenges and opportunities. International Journal Education Technologies High Education, 18, 16. https://doi.org/10.1186/s41239-021-00254-1

Cohen, M.D.; March, J.G.; Olsen, J.P. (1972). A garbage can model of organizational choice. Administrative Science Quarterly. 17, 1, 1-25. doi:10.2307/2392088. JSTOR 2392088

Hollands, F. M., \& Escueta, M. (2017). EdTech decision-making in higher education. Center for Benefit-Cost Studies of Education, Teachers College, Columbia University. Retrieved

from https://docs.wixstatic.com/ugd/cc7beb_39a11e93051142c8be0aa7a69d7eadee.pdf.

Iyengar, S. S., \& Lepper, M. R. (2000). When a choice is demotivating: Can one desire too much of a good thing? Journal of Personality and Social Psychology, 79,6, 995-1006. https://doi.org/10.1037/0022-3514.79.6.995

Ronghuai, H., Junfeng Y. \& Lanqin Z. (2013). The Components and Functions of Smart Learning Environments for Easy, Engaged and Effective Learning. International Journal for Educational Media and Technology, 7, 1, 4-14. 